\title{
EFFICACY OF THE NASOLABIAL FLAP IN RECONSTRUCTION OF SMALL POST-ABLATIVE DEFECTS OF THE OROFACIAL REGION
}

\author{
Manish Gupta1, Billola Umakanth Goud², Baswa Mani Kumari ${ }^{3}$ \\ ${ }^{1}$ Assistant Professor, Department of ENT, Osmania Medical College. \\ ${ }^{2}$ Associate Professor, Department of Surgical Oncology, MNJ Cancer Hospital/Regional Cancer Centre. \\ 3Professor, Department of Plastic Surgery, MNJ Cancer Hospital/Regional Cancer Centre.
}

\begin{abstract}
BACKGROUND

Resection of malignant lesions results in significant aesthetic and functional defects of the face and oral cavity. Reconstruction of large defects can be done with free and pedicled flaps. Because of bulk of pedicled flaps they may not give good cosmetic and functional results. Small-to-medium sized defects can be best managed by various local flaps. The Nasolabial flap is a simple flap used for the reconstruction of small-to-moderate orofacial defects created after the excision of primary malignant tumours, sometimes complimentary to other pedicled and free flaps.
\end{abstract}

\section{METHODS}

In this study, we did a retrospective analysis of 22 patients who underwent orofacial reconstruction with the nasolabial flap after excision of primary malignant lesions in MNJ Institute of Oncology and Regional Cancer Centre, Hyderabad, Telangana during 2010 to 2015. We have done both superiorly based and inferiorly based nasolabial flaps according to subsite involved.

\section{RESULTS}

Of the 22 patients, only 1 (4.5\%) patient had distal necrosis of a superiorly based flap which healed without any secondary procedures. All the remaining flaps healed uneventfully.

\section{CONCLUSION}

The Nasolabial Flap has continued to be a versatile and useful flap for reconstruction of small-to-medium defects of the or ofacial region.

\section{KEYWORDS}

Superiorly Based Nasolabial Flap, Inferiorly Based Nasolabial Flap, Orofacial Reconstruction.

HOW TO CITE THIS ARTICLE: Gupta M, Goud BU, Kumari BM. Efficacy of the nasolabial flap in reconstruction of small post-ablative defects of the orofacial region. J. Evolution Med. Dent. Sci. 2016;5(73):5359-5363, DOI: 10.14260/jemds/2016/1216

\section{BACKGROUND}

Resection of malignant lesions results in significant aesthetic and functional defects of the face and oral cavity. Reconstruction of large defects can be done with free and pedicled flaps. Small-to-medium sized defects can be best managed by various local flaps. Designed as a true myocutaneous flap based on the facial artery, the nasolabial flap is a robust and versatile flap. It is well suited to singlestage reconstruction of oral cavity defects or staged reconstructions of facial defects. The redundant skin extending from the medial canthus of the eye to the inferior margin of the mandible i.e. nasolabial sulcus and nasofacial groove is the donor site for the nasolabial flap. This area is relatively hairless except for the lower cheek in males, an important consideration in oral cavity reconstruction. The flap

First described in 600 A.D. in the Sushruta Samhita for correcting cutaneous defects, the Nasolabial Flap was used in 1868 by Thiersch for palatal intraoral reconstruction. Various modifications of the flap such as cutaneous and musculocutaneous along with or without a perforator have been practised as a successful surgical reconstructive option. The vascularity of the Nasolabial flap is based on the angular branch of the facial artery, the transverse facial artery and the infraorbital artery. This vascularity allows it to be either superiorly or inferiorly based. A superiorly based Nasolabial Flap can be used to reconstruct lower eyelid and medial cheek and nasal defects (Fig. 1a 1b 1c) and an inferiorly based flap for lip and intraoral defect. We have analysed retrospectively 28 Nasolabial Flap reconstructions in 22 patients with orofacial defects.
\end{abstract} itself is comprised of skin, subcutaneous tissue and the underlying musculature. The Nasolabial flap is a simple and easy reconstructive option for such defects with a high success rate.

Financial or Other, Competing Interest: None.

Submission 05-07-2016, Peer Review 26-08-2016,

Acceptance 02-09-2016, Published 10-09-2016.

Corresponding Author:

Dr. Manish Gupta,

H. No. 564, R. No. 12,

Banjara Hills, Hyderabad.

E-mail:drmanishgupta003@gmail.com

DOI: $10.14260 /$ jemds/2016/1216

\section{MATERIALS AND METHODS}

Twenty-two patients with post-excisional defects of the orofacial region underwent reconstruction with twenty-eight Nasolabial flaps between 2009 and 2014 in MNJ Institute of Oncology and Regional Cancer Centre, Hyderabad, Telangana. Medical records of these patients were reviewed. Site of reconstruction, type of flap superiorly based or inferiorly based and results in term of graft failure were analysed. Of the 22 patients, there were $12(54.5 \%)$ male and $8(36.4 \%)$ female patients. Of the 28 Nasolabial flaps, 5(17.8\%) were performed for the lower eyelids, 10(35.7\%) for the nose (Fig 2a, 2b, 2c), and $13(46.4 \%)$ for the oral cavity. Superiorly based flaps were 
planned for the eyelids, nose and the hard palate; inferiorly based flaps for the oral cavity except the hard palate. All reconstructions were single staged except one nasal reconstruction involving the columella, tip and mucosal lining of both alae, where bilateral nasolabial flaps were used. Bilateral flaps were used for 6 patients; two (33.33\%) for the nose, two $(33.33 \%)$ for the floor of the mouth, two (33.33\%) for the lower lips (Fig. 3a, 3b). Donor site is sutured with 4-0 Vicryl.

\section{Surgical Anatomy}

The subdermal plexus is supplied by feeder vessels from the branches of the facial artery and provides the blood supply to the nasolabial muscle and skin. This allows for high viability and allows thinning and shaping. The facial artery has four main branches in the face: the inferior labial artery, superior labial artery, alar artery and lateral nasal artery, and terminates as the angular artery. In majority of patients, the facial artery takes a medial course rather than a lateral course. The artery courses in the dense fibrous tissue at the oral commissure and continues along the superior border of the upper lip to the nasal ala. It then continues in a medial course along the nasofacial groove toward the medial canthus of the eye. Arterial perforators are concentrated in the inferior twothirds of the nasolabial groove. When raising a laterally pedicled flap, as is often the case in single-stage oral cavity defect reconstructions, incorporation of the lower one-third of the nasolabial groove is essential to ensure a robust vascular musculocutaneous base.

The relevant musculature is best understood as it relates to the facial artery. The facial artery is deep to the platysma, risorius, zygomaticus major and minor, levator labii superioris, and levator labii superioris alaeque nasi muscles. The artery is superficial to the mandible, buccinator muscle, and levator anguli oris muscle. The position of the artery deep to the majority of facial muscles suggests that the nasolabial flap may incorporate this muscle layer and be true musculocutaneous flap.

\section{TECHNIQUE}

All the procedures were performed under general anaesthesia. Wide local excision was followed by reconstruction; supraomohyoid neck dissection was done in 3 patients, two with floor of the mouth lesions and one with a lower alveolus lesion.

An inferiorly based flap was planned for the oral cavity reconstruction except for the hard palate. A fusiform shaped flap was marked in the nasolabial area limiting the medial border to the nasolabial fold. This was to achieve the final scar in the nasolabial fold without any dog-ears. The average dimensions of the flap were $8 \mathrm{~cm}$ in length and about $3 \mathrm{~cm}$ in breadth depending on the laxity of the available skin. The upper limit of the flap is inferior to the medial canthus of the eye along the nasolabial junction. The lower limit of the flap is one $\mathrm{cm}$ lateral to the oral commissure. The flap was elevated in the supramuscular plane. We routinely found a perforator at the level of the oral commissure at which level the base is narrowed safely. A transbuccal tunnel wide enough to admit two fingers was made adjacent to the oral defect. The base of the flap is de-epithelised; transferred to the defect in the oral cavity through the transbuccal tunnel. The final inset of the flap was done with 4-0 Monocryl. Undermining of donor site is done if required. Primary closure is done in layers.

Superiorly based flaps were planned for the lower eyelids and nose. The flap dimensions are usually dependent upon the size of the defect. The average size of the eyelid defects were $5 \times 3 \mathrm{~cm}$ involving the skin and orbicularis oculi muscle. The lid margin and the tarsal plate were spared in all the cases. The flaps were based just below the medial canthal level, rotated into the defect and primary closure of the donor area was done.

We did single stage reconstruction for all the alar defects of the nose, sometimes excising the minimal bridge of the skin between the defect and the nasolabial groove, rotating the flap into the defect. But the disadvantage was obliteration of the nasolabial groove and the natural supra alar concavity. For one case of total nose reconstruction, we used bilateral nasolabial flaps for mucosal lining and the median forehead flap was used for the cover. The bases of the nasolabial flaps were deepithelised and the flaps were twisted 180 degrees to form the nasal lining. A second stage was needed for this patient for division and final inset of the forehead flap. We did a twostaged reconstruction with nasolabial flaps for a defect involving the columella, nasal tip and mucosal lining of both alae. Flap division and final inset was done after 3 weeks.

\section{RESULTS}

Of the 22 patients, there were $12(54.5 \%)$ male and $8(36.4 \%)$ female patients. Of the 28 Nasolabial flaps, 5(17.8\%) were performed for the lower eyelids, $10(35.7 \%)$ for the nose (Fig. $2 \mathrm{a}, 2 \mathrm{~b}, 2 \mathrm{c})$, and $13(46.4 \%)$ for the oral cavity. Superiorly based flaps were planned for the eyelids, nose and the hard palate; inferiorly based flaps for the oral cavity except the hard palate. All reconstructions were single staged except one nasal reconstruction involving the columella, tip and mucosal lining of both alae, where bilateral nasolabial flaps were used. Bilateral flaps were used for 6 patients; two (33.33\%) for the nose, two (33.33\%) for the floor of the mouth, two (33.33\%) for the lower lips (Fig. 3a, 3b). Of the 22 patients, only 1 (4.5\%) patient had distal necrosis of a superiorly based flap which healed without any secondary procedures. All the remaining flaps healed uneventfully.

Post-Operative Images

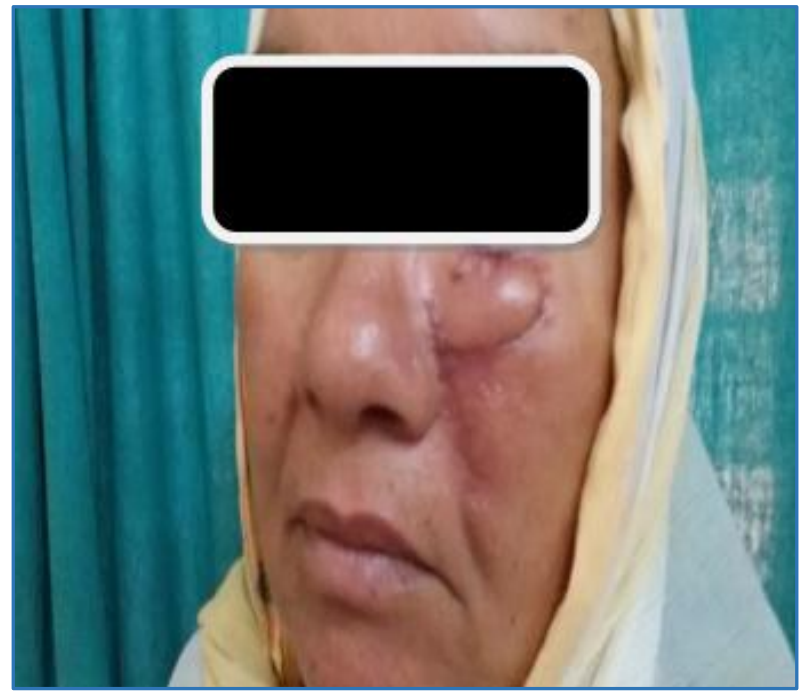

Fig. 1: Post-operative Picture of a Superiorly based Nasolabial Flap for Defect in Lower Eyelid 


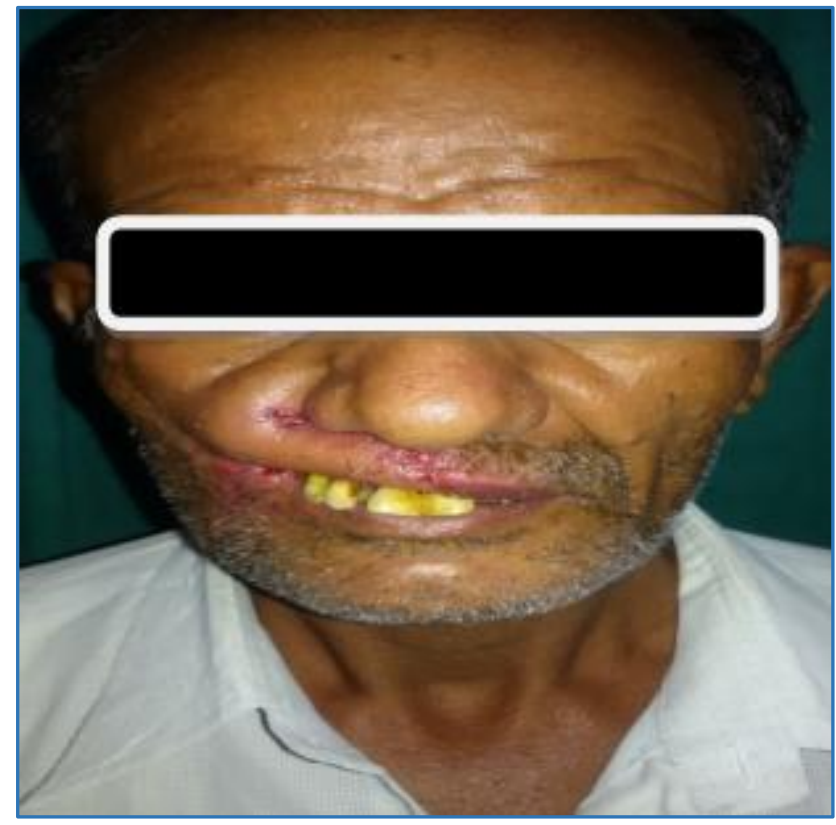

Fig. 2: Post-operative picture of a Superiorly based Nasolabial Flap for Defect in Upper Lip

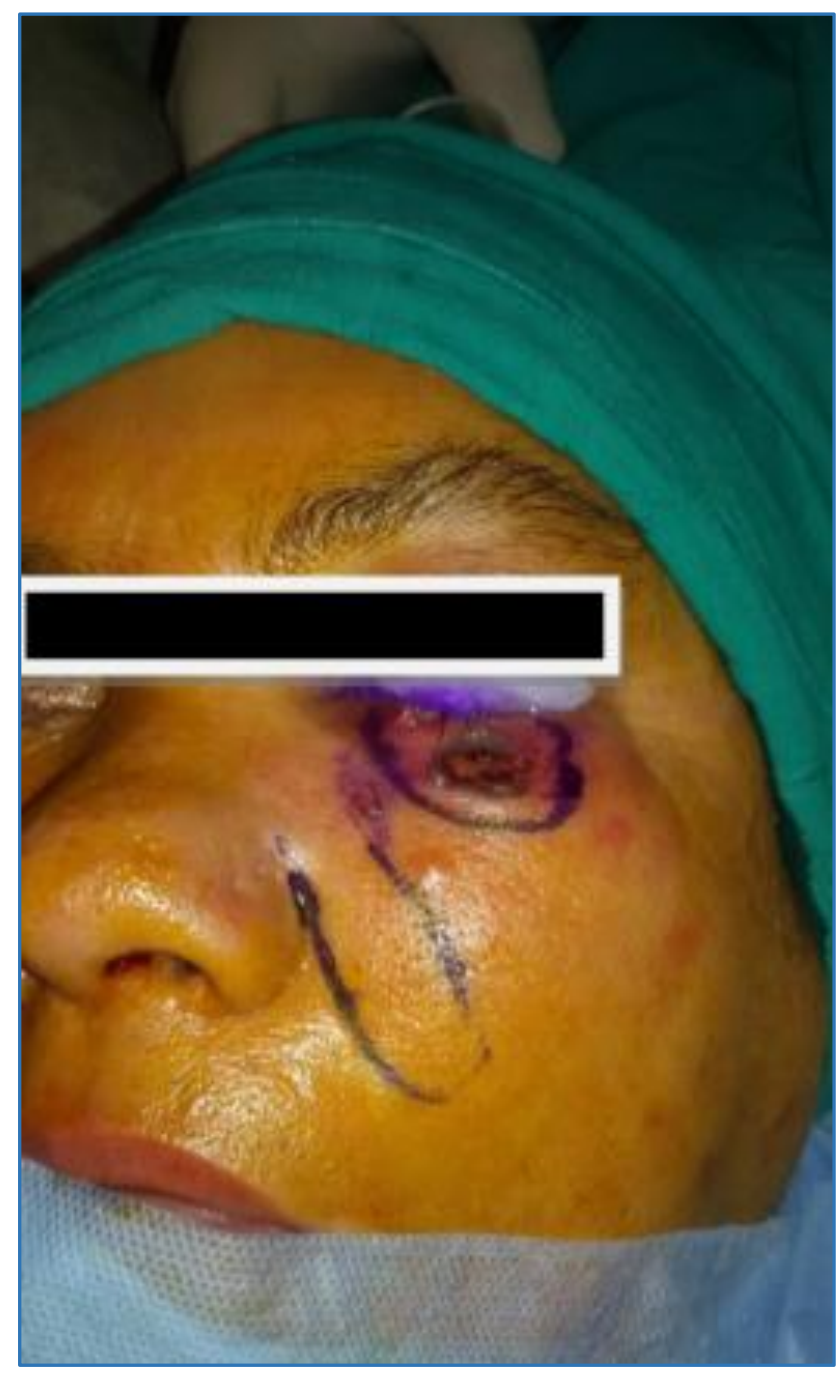

Fig. 3: Marking of Superiorly based Nasolabial Flap for the Lesion of the Lower Lid

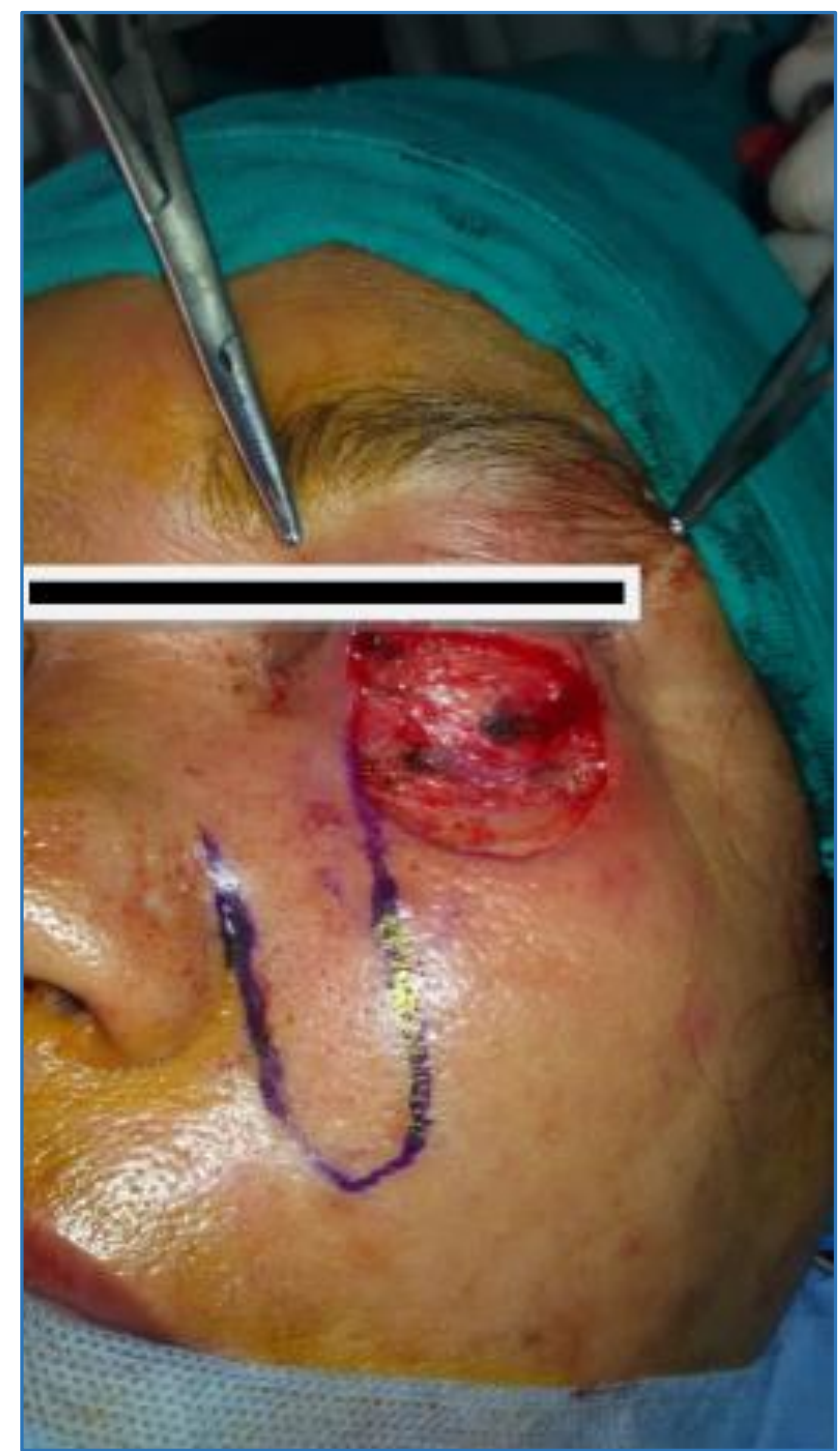

Fig. 4: Picture of Defect after Excision of the Lesion

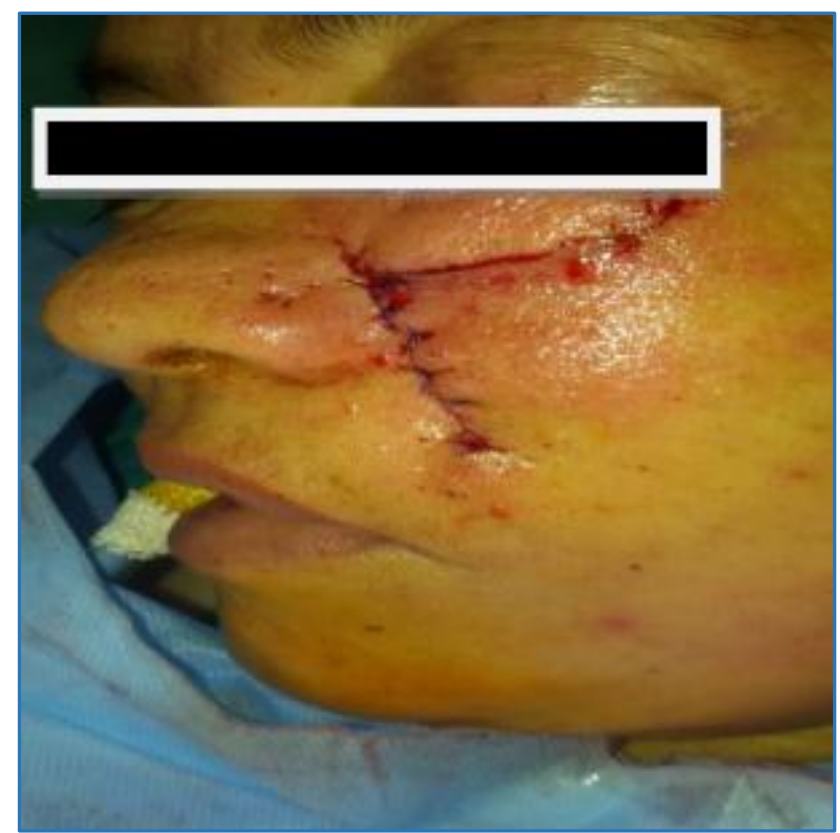

Fig. 5: Image of completed Superiorly based Nasolabial Flap 


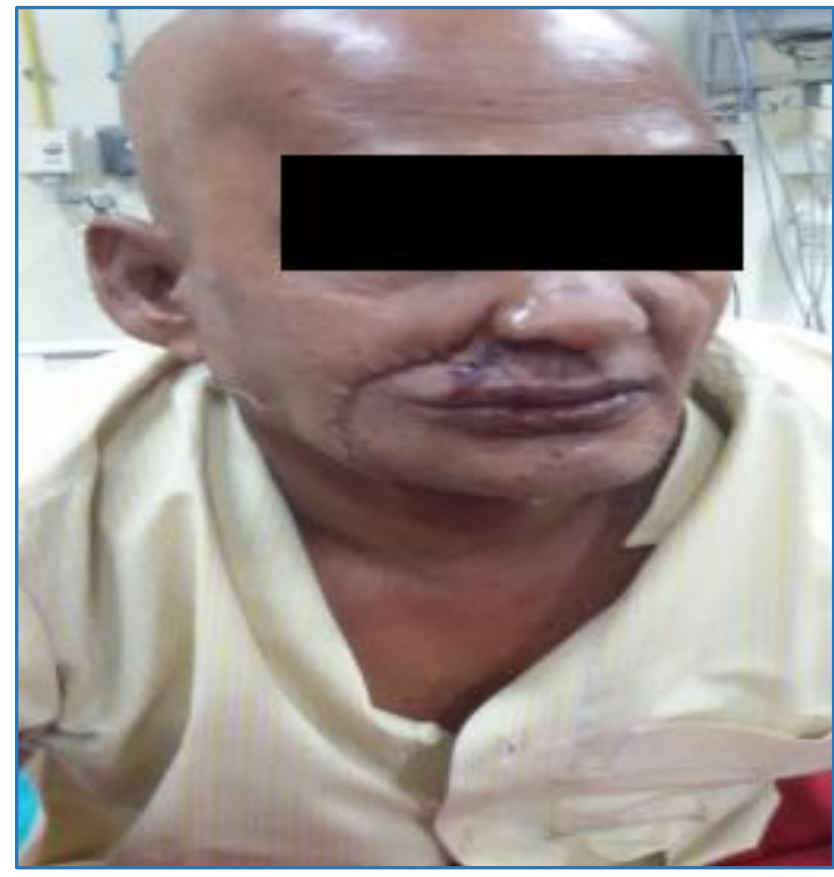

Fig. 6: Post-operative Image of healed Inferiorly based Nasolabial Flap for Lesion of the Upper Lip

\begin{tabular}{|c|c|c|c|c|c|c|}
\hline $\begin{array}{c}\text { Sl. } \\
\text { No. }\end{array}$ & Site & $\begin{array}{c}\text { Type of } \\
\text { Malignancy }\end{array}$ & Grade & $\begin{array}{c}\text { Mean } \\
\text { Size } \\
\text { of } \\
\text { Tumour }\end{array}$ & $\begin{array}{c}\text { Margin } \\
\text { Given }\end{array}$ & $\begin{array}{c}\text { Mean } \\
\text { Size } \\
\text { of } \\
\text { Defect }\end{array}$ \\
\hline 1 & $\begin{array}{c}\text { Lower } \\
\text { Eye } \\
\text { Lids }\end{array}$ & $\begin{array}{c}\text { Basal Cell } \\
\text { Carcinoma }\end{array}$ & $2.4 \mathrm{~cm}$ & $0.5 \mathrm{~cm}$ & $2.9 \mathrm{~cm}$ \\
\hline 2 & Nose & $\begin{array}{c}\text { Basal Cell } \\
\text { Carcinoma }\end{array}$ & & $2.4 \mathrm{~cm}$ & $0.5 \mathrm{~cm}$ & $2.9 \mathrm{~cm}$ \\
\hline 3 & $\begin{array}{c}\text { Oral } \\
\text { Cavity }\end{array}$ & $\begin{array}{c}\text { Squamous } \\
\text { Cell } \\
\text { Carcinoma }\end{array}$ & $\begin{array}{c}\text { Moderately } \\
\text { Differentiated }\end{array}$ & $3.6 \mathrm{~cm}$ & $1 \mathrm{~cm}$ & $4.6 \mathrm{~cm}$ \\
\hline
\end{tabular}

\section{DISCUSSION}

Orofacial defects created after wide local excision of cancers need reconstruction to restore the function and symmetry. The location, size and depth of the defect determine the reconstructive modality of choice.

Medium and large composite defects require major microvascular reconstructions or sometimes the distant pedicled pectoralis major and deltopectoral flaps depending on the expertise and infrastructure available. Small and superficial defects may be covered with skin grafts but their tendency to contract, a colour mismatch and the requirement for immediate postoperative radiotherapy for some patients make skin grafts a less preferred choice of reconstruction. A simple and effective reconstruction of the small defects should be ideally planned with local flaps with minimal donor site morbidity. Nasolabial flaps are very useful tools in such a situation.

Our purpose in this paper is to share our experience with the outcome of Nasolabial flap reconstruction in small defects of the orofacial region caused after wide local excision of orofacial malignancies.

Dieffenbach first described the use of a nasolabial flap in 1830, where superiorly based nasolabial flaps were used to reconstruct nasal alae. Simultaneous use of the nasolabial flap by Von Langenbeck in 1864 and Esser in 1921 have been described.
Twenty-two patients underwent Nasolabial flap reconstructions of the orofacial region in our study. The location of the facial artery beneath the facial kinetic muscles allows the development of the nasolabial flap.[1]

In our study, 3 patients underwent wide local excision of the primary tumour along with supraomohyoid neck dissection. The facial artery was ligated in these patients. This never affected the viability of the flap probably due to the rich subdermal vascular plexus.[2] This flap has advantages in colour match, location, excellent blood supply, and absence of hair and can be easily transferred to the defect with local anaesthesia. Minimal donor site deformity has been reported due to the final scar in the natural crease at the nasolabial fold. [3] Nasolabial flaps have long been used by several authors for the reconstruction of facial defects.

Lazardis et al in 1998 successfully used inferiorly and superiorly based nasolabial flaps for reconstruction of moderate-sized oronasal defects over a ten-year period with minimal complications. ${ }^{[4]}$

Hofstra et al in 2000 confirmed good functional and aesthetic outcome following intraoral reconstruction of small defects with nasolabial flaps.[5] Van Wijk et al in 2000 used 105 nasolabial flaps in 79 patients for reconstruction of defects of the anterior floor of the mouth. They observed that $82 \%$ of the flaps healed successfully; flap survival was $95 \%$; considerable flap loss occurred in 5\%; primary dehiscence was observed in $5 \%$ of all flaps, which healed spontaneously with conservative treatment. They concluded that a lengthy and complex reconstruction is always not needed for small intraoral defects and the nasolabial flap offers a safe and simple alternative.[6]

The largest publication of nasolabial flaps was by Varghese et al in 2001 of their series of 224 cases in which more complications were reported in post-irradiated cases than in primary $(\mathrm{p}=0.03) .[7]$

Maurer et al in 2002, in their series of 26 patients reported that 23 patients $(88 \%)$ underwent successful prosthetic rehabilitation after reconstruction of defects about the size of $5 / 5 \mathrm{~cm}$ caused due to resection of squamous cell carcinoma of the anterior floor of the mouth. They concluded that the nasolabial flap represents an aesthetic and functional alternative to microvascular reconstruction, especially in the older, medically compromised patients who are a poor surgical risk.[8]

Navarro et al successfully described a reconstructive technique in 12 oncologic patients who underwent segmental mandibulectomy with soft tissue resection. Reconstruction of the mandible was done with an iliac crest flap combined with a nasolabial flap for intraoral soft tissue and immediate placement of osseointegrated implants. [9]

A total of 28 nasolabial flap reconstructions were performed in our study. Assessment of the depth and location of the defect is important in planning the type of nasolabial flap. Facial defects comprising the medial cheek, nose, lower eyelids and intraoral defects of about $5 \mathrm{~cm}$ wide or less are best restored with the Nasolabial flap.

Small defects of the floor of the mouth often require reconstruction for free tongue mobility and function. By using bilateral inferior based nasolabial flaps in our patients, we were able to significantly improve the functional outcome and speech. In our study, a superiorly based nasolabial flap was used for reconstruction in five patients who had lower eye lid defects and ten patients with nasal defects. 
Good results particularly for nasal reconstruction have been achieved with the superiorly based nasolabial flap. ${ }^{[10]}$ Primary closure, covering the defects with split thickness skin grafts and secondary healing are some of the reconstructive options available for small intraoral defects, which may result in speech and swallowing problems.

\section{CONCLUSION}

Reconstruction with a nasolabial flap is simple and predictable and minimises the problems related to speech and swallowing.

With our experience we conclude that nasolabial flap is a simple flap, extremely useful for small defects of the orofacial region, safer even in post-irradiated patients with minimal complications.

\section{REFERENCES}

1. Hagan WE, Walker LB. The nasolabial musculocutaneous flap: clinical and anatomical correlations. Laryngoscope 1998;98(3):341-6.

2. Hynes B, Boyd JB. The nasolabial flap. Axial or random? Arch Otolaryngol Head Neck Surg 1988;114(12):138991.

3. Cameron RR, Latham WD, Dowling JA. Reconstructions of the nose and upper lip with nasolabial flaps. Plast Reconstr Surg 1973;52(2):145-50.
4. Lazardis N, Zouloumis L, Venetis G, et al. The inferiorly and superiorly based nasolabial flap for the reconstruction of moderate-sized oronasal defects. J Oral Maxillofac Surg 1998;56(11):1255-9, discussion 1260.

5. Hofstra EI, Hofer SO, Nauta JM, et al. Oral functional outcome after intraoral reconstruction with nasolabial flaps. Br J Plast Surg 2004;57(2):150-5.

6. Van Wijk MP, Damen A, Nauta JM, et al. Reconstruction of the anterior floor of the mouth with the inferiorly based nasolabial flap. European Journal of Plastic Surgery 2000;23(4):200-3.

7. Varghese BT, Sebastian P, Cherian T, et al. Nasolabial flaps in oral reconstruction: an analysis of 224 cases. Br J Plast Surg 2001;54(6):499-503.

8. Maurer P, Eckert AW, Otto C, et al. Reconstruction of the anterior floor of the mouth with nasolabial flaps. Report of 10 years' experience. Schweiz Monatsschr Zahnmed 2002;112(5):463-6.

9. Cuellar NC, Caicoya SJ, Sanz AJJ, et al. Mandibular reconstruction with iliac crest free flap, nasolabial flap, and osseointegrated implants. J Oral Maxillofac Surg 2014;72(6):1226. e1-15.

10. Rohrich RJ, Conrad MH. The superiorly based nasolabial flap for simultaneous alar and cheek reconstruction. Plast Reconstr Surg 2001;108(6):1727-30, quiz 1731. 